\section{Award winning extraoral imaging}

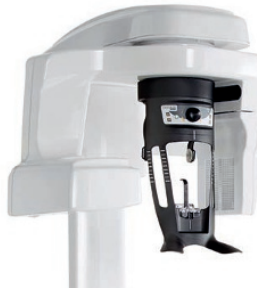

Carestream Dental is delighted to announce that its CS 8100SC 3D extraoral imaging system has received a Bronze accolade in the Edison Award 2018 within the Dental/Medical Digital Imaging category.

The prestigious award is inspired by Thomas Edison's persistence and inventiveness, highlighting innovation, creativity and ingenuity of solutions across the globe. Nominated technologies are judged by more than 3,000 senior business executives and academics based on their concept, value, impact and delivery.

The CS 8100SC 3D is a fine example of Carestream Dental's commitment to developing innovations that streamline workflows and ensure the highest standard of diagnostics. It offers $3 \mathrm{D}$ panoramic, cephalometric and $\mathrm{CBCT}$ imaging, as well as impression scanning to cater to all the needs of the modern dental practice.

Its minimal footprint and affordability brings high resolution imaging within the reach of every business, facilitating delivery of enhanced patient care. The CS 8100SC 3D also maximises safety with a low dose programme delivering $3 \mathrm{D}$ imaging at a radiation dose that is lower than or equal to panoramic imaging.

For more information please contact Carestream Dental on 08001699692 or visit www.carestreamdental.co.uk

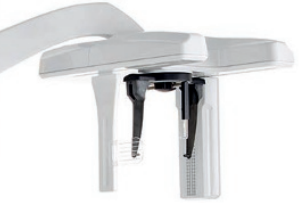

\section{Orthodontic brackets you can rely on}

The Victory Series Active Self-Ligating Brackets from 3M Oral Care offer an array of advantages for all orthodontic specialists to utilise.

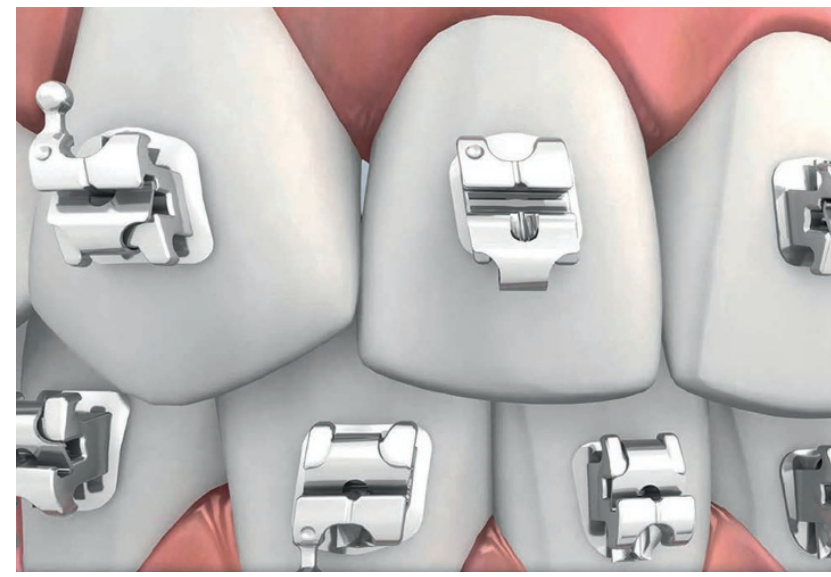

The heat treated, nickel-cobalt brackets provide secure and robust ligation, while the rounded edges of the precise MIM bracket bodies reduce archwire binding.

Improving reliability of treatment, the brackets feature microetched, anatomically curved mesh pads to provide greater bond strength and are made from 17-4 PH stainless steel for higher resistance against deformation. Patient comfort is optimised through low profile hooks on each bracket.

Find out how you and your patients could benefit from the Victory Series Active Self-Ligating Brackets from 3M Oral Care today: for more information, call 08458734066 or visit http://solutions.3m. co.uk/wps/portal/3M/en_GB/orthodontics_EU/Unitek/

\section{$3 \mathrm{M}$ and Victory Series are trademarks of the 3M Company.}

\section{A definitive fixed solution}

Do you want to offer a fixed solution for a wider variety of fully edentulous patients? The Trefoil system from Nobel Biocare is the key to providing even more patients with a solution they can trust.

Using an innovative self-adjusting fixation mechanism and just three implants, the Trefoil system has made fitting a premanufactured bar possible for the first time. This allows you to offer a long-lasting solution to patients who were unable to receive this type of treatment before.

The Trefoil system provides patients with benefits such as:

- A higher bite force

- Better retention and stability

- A better quality of life.

Furthermore, the Trefoil system also holds a number of benefits over removable prosthetic solutions. Patients won't experience mucosal problems and wear to the components that often cause difficulties for those with removable dentures.

The combination of the pre-manufactured titanium bar, three implants, simplified workflow and smaller amount of restorative components leads to a faster time-to-teeth for patients. Now you

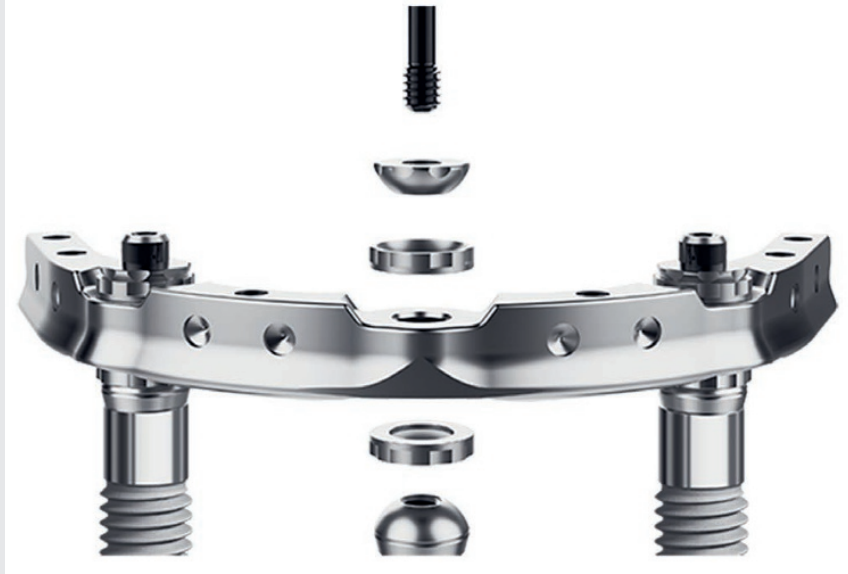

can offer a solution that will return patients to a better standard of living, fast.

For more information, contact Nobel Biocare on 0208756 3300, or visit www.nobelbiocare.com/uk/en/trefoil. 